\title{
A SIMPLE PROOF OF THE FUNDAMENTAL THEOREM OF KIRBY CALCULUS ON LINKS
}

\author{
NING LU
}

\begin{abstract}
In this paper, we relate surgeries on links and Heegaard decompositions, relate framed links and surface mapping classes, and give a simple proof of the fundamental theorem of Kirby calculus on links by the presentation of the surface mapping class groups.
\end{abstract}

Surgery on links, a beautiful way to describe 3-manifolds, was discovered independently by Wallace [Wa] and Lickorish [Li] in the early sixties. It has become even more attractive since Kirby found a criterion-so called Kirby calculus today - to tell how two such surgeries which define the same 3-manifold are related by some combinatorial moves. In Kirby's original proof Cerf theory played a central role which was not easily understood. A more intuitive proof was suggested by Hatcher-Thurston-Wajnryb's explicit presentation [W] of the surface mapping class groups. In this paper, we are going to give a new proof of the fundamental theorem of Kirby calculus using this idea. Instead of proving Kirby's original version, we will prove directly the simpler version of FennRourke [FR]:

Theorem 1.1 (Kirby-Fenn-Rourke). Two integer framed links determine the same 3-manifold if and only if they are related by $K^{ \pm 1}$-moves. Moreover, if all components of these two links are unknotted, then all $K^{ \pm 1}$-moves can be chosen such that all links involved in the process have unknotted components.

A one-page proof of the equivalence of these two versions can be found in the paper [FR].

A $K$-move, as pictured in Figure 1.1, is defined to be an elimination of a \pm 1 labeled unknotted component of the link by doing the corresponding surgery. And a $K^{-1}$-move is the reverse process of such a $K$-move.

A nice thing in our proof is that one actually can see how Heegaard decomposition-another important way to describe 3-manifold-and surgery on links can be transformed to each other. In addition, if two framed links determine the same 3-manifold our proof gives an algorithm for relating them by $K^{ \pm 1}$ moves.

Received by the editors February 1, 1990.

1980 Mathematics Subject Classification (1985 Revision). Primary 57M25, 57N10. 

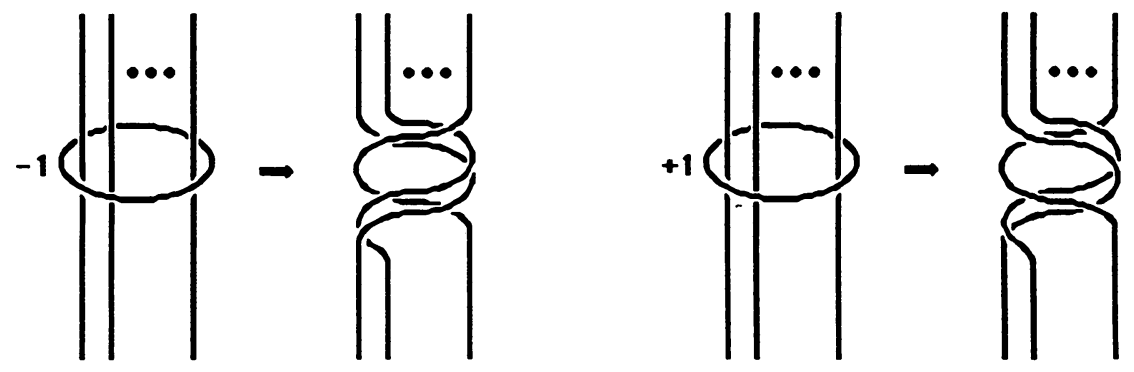

FIGURE 1

\section{HeegaARd decompositions of the 3-MANifold ASSOCIATED TO A FRAMED LINK}

The Heegaard decomposition and the surgery on links are two important methods to describe 3-manifolds; they can be translated from one to the other easily by using the Lickorish's Dehn twist generators of the surface mapping class groups.

Given a framed link, we are going to construct a Heegaard decomposition of the 3-manifold associated to it.

Let $\mathbf{L}$ be a $P L$-link in $\mathbf{S}^{3}$ with $r$ components. Let $X=\mathbf{S}^{3}-N(\mathbf{L})$ be the complement space of $\mathbf{L}$. Let $\left\{\lambda_{j}, \mu_{j}\right\}_{j=1, \ldots, r}$ be the fixed pairs of simple closed curves on the boundary components $T_{j}$ 's of the boundary $\partial X$ of $X$, such that, for each $j=1, \ldots, r, \lambda_{j}$ is a longitude circle of the solid handlebody $N\left(\mathbf{L}_{j}\right), \mu_{j}$ a meridian circle of $N\left(\mathbf{L}_{j}\right)$, and $\lambda_{j}$ is null-homologous in the knot complement $\mathbf{S}^{3}-N\left(\mathbf{L}_{j}\right)$.

For $\left\{\left(p_{j}, q_{j}\right)\right\}$ a system of pairs of coprime integers, the closed 3-manifold associated to the framed link $\left(\mathbf{L},\left\{\left(p_{j}, q_{j}\right)\right\}\right)$, denoted by $M\left(\mathbf{L},\left\{\left(p_{j} / q_{j}\right)\right\}\right)$, is defined to be the 3-manifold obtained from $X$ by sewing a solid torus along each $T_{j}$ with the meridian slope $\lambda_{j}^{q_{j}} \mu_{j}^{p_{j}}$.

When $q_{j}=1$, for all $j=1, \ldots, r$, we call the surgeries integer surgeries, and we will simply denote by $M(\mathbf{L})=M\left(\mathbf{L},\left\{p_{j}\right\}\right)=M\left(\mathbf{L},\left\{p_{j} / 1\right\}\right)$. In this section, we study only the integer framed links. In general, a rational framed link can be changed into an integer framed link according to Rolfsen's work [R].

Instead of Theorem 1.1, we will show the following equivalent theorem.

Theorem 2. Let $\mathbf{L}$ and $\mathbf{L}^{\prime}$ be two integers framed links in $\mathbf{S}^{3}$. They determine the same 3-manifold $M(\mathbf{L})=M\left(\mathbf{L}^{\prime}\right)$ if and only if there is a finite sequence

$$
\mathbf{L}=\mathbf{L}^{0}, \mathbf{L}^{1}, \ldots, \mathbf{L}^{n}=\mathbf{L}^{\prime},
$$

such that, for $i=1, \ldots, n, \mathbf{L}^{i}$ is obtained from $\mathbf{L}^{i-1}$ by one of the following steps:

(I) $D$ (isk)-move. Add an unknotted component which bounds a disk in $X^{i-1}=$ $\mathbf{S}^{3}-\mathbf{L}^{i-1}$ to $\mathbf{L}^{i-1}$ with the surgery label \pm 1 (Figure 2).

(II) A(nnulus)-move. Add two unknotted components which bound an untwisted annulus in $X^{i-1}$ of $\mathbf{L}^{i-1}$ with the surgery labels one +1 and the other -1 , (Figure 3).

(III) $K$ (irby)-move. Eliminate an unknotted component with label \pm 1 of $\mathbf{L}^{i-1}$ by doing the corresponding surgery in $\mathbf{S}^{3}$ (Figure 1). 


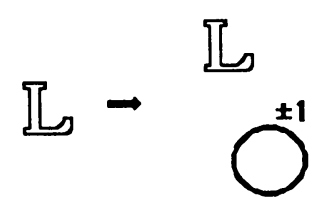

FIGURE 2

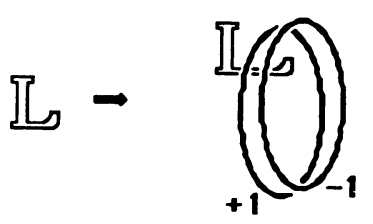

FIGURE 3

Obviously, a $K^{-1}$-move is a composition of an $A$-move, since we may insert two copies of that component for $K^{-1}$-move with one sign +1 and one -1 by an $A$-move, then use a $K$-move to eliminate one of them. A $D$-move is actually a $K^{-1}$ move. And an $A$-move is a composition of two $K^{-1}$-moves. Therefore, Theorem 1 and Theorem 2 are equivalent.

Let $\mathbf{F}_{g}$ be an unknotted embedding of the closed orientable surface of genus $g$ in $\mathbf{S}^{3}$ which bounds two handlebodies $\mathbf{H}_{g}$ and $\mathbf{H}_{g}^{\prime}$, let $O$ be a fixed basepoint in $\mathbf{F}_{g}$, and let $\mathscr{B}=\left\{a_{1}, b_{1}, a_{2}, b_{2}, \ldots, a_{g}, b_{g}\right\}$ be a chosen system of basecurves on the surface $\mathbf{F}_{g}$ based at $O$, such that $a_{i}$ 's are meridians of the handlebody $\mathbf{H}_{g}$, and the $b_{i}$ 's are meridians of the handlebody $\mathbf{H}_{g}^{\prime}$.

A Heegaard decomposition of 3-manifold can be described by an orientationpreserving self-homeomorphism of the surface $\mathbf{F}_{g}$ up to isotopy, i.e. an element of the mapping class group $\mathscr{M}_{G}$ of the surface $\mathbf{F}_{g}$. Given a mapping class $f$ of $\mathscr{M}_{g}$, the associated 3-manifold $M(f)$ is defined to be the 3-manifold obtained by regluing the handlebodies $\mathbf{H}_{g}^{\prime}$ and $\mathbf{H}_{g}$ by identifying the points $x$ of $\partial \mathbf{H}_{g}^{\prime}$ to the points $(x) f$ of $\partial \mathbf{H}_{g}$.

We embed the link $\mathbf{L}$ in the surface $\mathbf{F}_{g}$ (for $g$ large enough, e.g. $g \geq$ $b(\mathbf{L})$ the bridge number of $\mathbf{L})$. For each component $\mathbf{L}_{j}$ of $\mathbf{L}$, the slope of a component of $T_{j} \cap \mathbf{F}_{g}$ in $T_{j}$ has an expression $\lambda_{j} \mu_{j}^{s_{j}}$, for some integer $s_{j}$. The number $s_{j}$ is called the embedding index of $\mathbf{L}_{j}$. Denote by $\Delta_{j}^{ \pm 1}$ the Dehn twist of the circle given by the component $\mathbf{L}_{j}$ in $\mathbf{F}_{g}$; we have that

\section{Proposition 3.}

$$
M\left(\Delta_{j}^{ \pm 1}\right)=M\left(\mathbf{L}_{j}, s_{j} \pm 1\right) .
$$

Proof. By the definition, $M\left(\Delta_{j}\right)$ is obtained by splitting $\mathbf{F}_{g}$ in $\mathbf{S}^{3}$ and regluing according to the map $\Delta_{j}$ which changes $\mathbf{S}^{3}$ only a neighborhood $N\left(\mathbf{L}_{j}\right)$ of the twist curve given by $\mathbf{L}_{j}$. Thus $M\left(\Delta_{j}\right)$ can be also obtained by doing some surgery along the component $\mathbf{L}_{j}$. Consider the way two copies of the annulus $\mathbf{F}_{g} \cap N\left(\mathbf{L}_{j}\right)$ are glued, it is equivalent to doing a surgery on $\mathbf{L}_{j}$ by replacing the neighborhood $N\left(\mathbf{L}_{j}\right)$ by a solid torus whose meridian circle intersects each of the components of $\mu_{j}$ and $T_{j} \cap \mathbf{F}_{g}$ at one point (Figure 4). Thus, the surgery is one of the $\left(s_{j} \pm 1\right)$-surgeries. By convention, we use the same sign for both the Dehn twist and the surgery, namely the $\left(s_{j}+1\right)$-surgery for $\mathbf{L}_{j}$ of $\Delta_{j}$, and the $\left(s_{j}-1\right)$-surgery of $\mathbf{L}_{j}$ for $\Delta_{j}^{-1}$.

For genus $g$ large enough, we may assume the embedding has the property that, for each component $\mathbf{L}_{j}$ of $\mathbf{L}$, there is some $1 \leq i \leq g$ such that the intersection $\mathbf{L} \cap b_{i}=\mathbf{L}_{j} \cap b_{i}$ is one point. Given any framed link $\mathbf{L}=\left(\mathbf{L}_{j}, p_{j}\right)$, the next proposition will tell us that the link $\mathbf{L}$ can be embedded in the surface $\mathbf{F}_{g}$ so that the embedding index of the component $\mathbf{L}_{j}$ is $p_{j}-1$, for all $j$. 

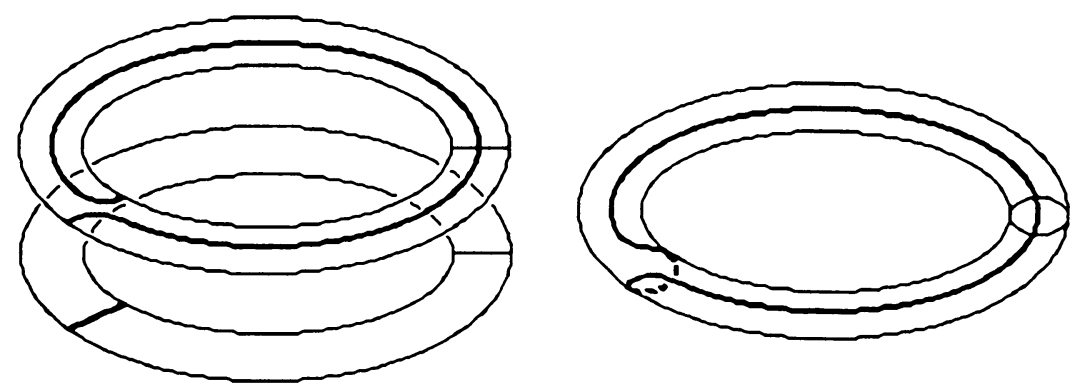

FIGURE 4
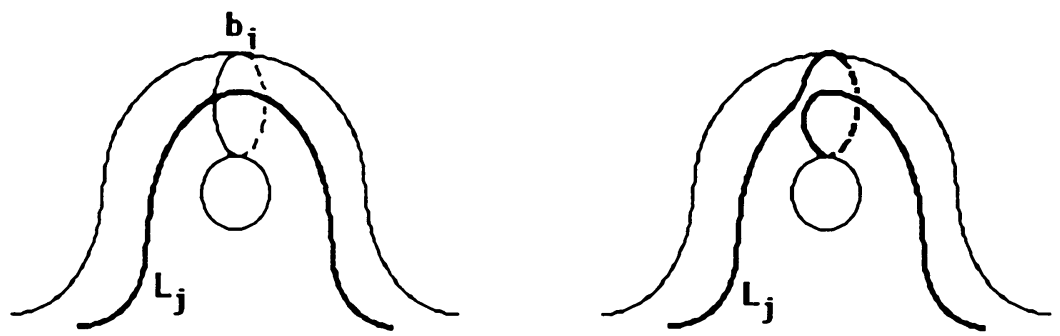

FIGURE 5

Proposition 4. Given an embedding of the knot $\mathbf{L}_{j}$ in $\mathbf{F}_{g}$ with the embedding index $s_{j}$, if $\mathbf{L}_{j} \cap b_{i}$ is one point, for some $i \in\{1, \ldots, g\}$, we may reembed $\mathbf{L}_{j}$ in $\mathbf{F}_{g}$ by twisting around $b_{i}$ once to get an embedding of $\mathbf{L}_{j}$ with the embedding index either $s_{j}+1$ or $s_{j}-1$, depending on the twisting orientation.

Proof. As pictured in Figure 5, the proposition is clear by comparing the slopes $T_{j} \cap \mathbf{F}_{g}$ before and after twisting the handle $b_{i}$.

Corollary 5. A closed 3-manifold is obtained by some integer surgeries on the link $\mathbf{L}$, if and only if it has a Heegaard decomposition described by a composition $\varphi$ of Dehn twists of some disjoint, simple, closed curves, which is isotopic to the link $\mathbf{L}$, in some surface $\mathbf{F}_{g}$ unknottedly embedded in $\mathbf{S}^{3}$.

\section{REPRESENTING SURFACE MAPPING CLASSES BY FRAMED LINKS}

Given a Heegaard decomposition of a 3-manifold $M$, it can be described by an element $\varphi$ of the mapping class group $\mathscr{M}_{g}$ of the closed orientable surface $\mathbf{F}_{g}$. Certainly $\varphi$ can be written as a composition of the Lickorish generators of the group $\mathscr{M}_{g}$. Denote the Lickorish generators by $\Lambda_{1}, \Lambda_{2}, \ldots, \Lambda_{r}$ which are Dehn twists of the simple closed curves $\lambda_{1}, \lambda_{2}, \ldots, \lambda_{r}$. Fixing a regular neighborhood $\mathbf{F}_{g} \times[0,1]$ of the closed surface $\mathbf{F}_{g}$, choosing a bunch of parallel copies of the surface $\mathbf{F}_{g} \times \varepsilon_{1}, \mathbf{F}_{g} \times \varepsilon_{2}, \ldots, \mathbf{F}_{g} \times \varepsilon_{r}$, for some $0<\varepsilon_{1}<\varepsilon_{2}<$ $\varepsilon_{r}<1$, and embedding those curves one by one in the copies of the surface in the composition order, we obtain a link

$$
\mathbf{L}=\left\{\left(\lambda_{j}, \varepsilon_{j}\right)\right\}_{j=1, \ldots, r} .
$$

Because all the $\lambda_{j}$ 's are given by the Lickorish generators, the imbedding indices are all 0 . Thus each Dehn twist is equivalent to a \pm 1 -surgery on the 
corresponding curve, and there is a unique way to choose the label either +1 or -1 for each component of the link according to the twisting orientation. Obviously, the surgery on this framed link gives the same 3-manifold as the Heegaard decomposition does.

In general, consider the same closed orientable surface $\mathbf{F}_{g}$ in the 3-sphere $\mathbf{S}^{3}$ as in the last section. Denote by $\mathbf{F}_{g} \times[0,1]$ a regular neighborhood of $\mathbf{F}_{g}$, such that $\mathbf{F}_{g} \times 0 \subset \mathbf{H}_{g}$ and $\mathbf{F}_{g} \times 1 \subset \mathbf{H}_{g}^{\prime}$. Let $\varepsilon_{0}=0<\varepsilon_{1}<\varepsilon_{2}<\varepsilon_{r}<$ $\varepsilon_{r+1}=1$ be a sequence of numbers, called level numbers. Let $f_{1}, f_{2}$ and $f_{r}$ be a sequence of mapping classes of $\mathscr{M}_{g}$. Denote by $M\left(f_{1}, f_{2}, \ldots, f_{r}\right)$ the 3-manifold obtained by gluing together the handlebodies $\mathbf{H}_{g}, \mathbf{H}_{g}^{\prime}$ and thicken surfaces $\mathbf{F}_{g} \times\left[\varepsilon_{j}, \varepsilon_{j+1}\right]$, for $j=1,2, \ldots, r$ along there boundary surfaces in the way that, the boundary surface of $\mathbf{H}_{g}$ and that of $\mathbf{H}_{g}^{\prime}$ are identified to the boundary surfaces $\mathbf{F}_{g} \times 0$ of $\mathbf{F}_{g} \times\left[0, \varepsilon_{1}\right]$ and $\mathbf{F}_{g} \times 1$ of $\mathbf{F}_{g} \times\left[\varepsilon_{r}, 1\right]$ respectively by the identities, and the points $x$ of the boundary surface $\mathbf{F}_{g} \times j$ of the thickens surface $\mathbf{F}_{g} \times\left[\varepsilon_{j}, \varepsilon_{j+1}\right]$ are identified to the points $(x) f$ of the boundary surface $\mathbf{F}_{g} \times j$ of $\mathbf{F}_{g} \times\left[\varepsilon_{j-1}, \varepsilon_{j}\right]$, for $j=1,2, \ldots, r$. From the construction, clearly the 3-manifold $M\left(f_{1}, f_{2}, \ldots, f_{r}\right)$ is homeomorphic to the 3-manifold $M\left(f_{r} \cdots f_{2} f_{1}\right)$.

Therefore, given a framed link $\mathbf{L}=\left\{\mathbf{L}_{j}\right\}_{1 \leq j \leq r}$, if we may embed the link in the collection of surfaces $\mathbf{F}_{g} \times\left\{\varepsilon_{1}, \varepsilon_{2}, \ldots, \varepsilon_{r}\right\}$ with proper embedding indices, such that the component $\mathbf{L}_{j}$ is embedded as the circle $\left(\theta_{j}, \varepsilon_{j}\right)$, and the surgery along $\mathbf{L}_{j}$ with the given coefficient is equivalent to the Dehn twist $\Theta_{j}$ of the circle $\theta_{j}$, then we will say that the link $\mathbf{L}$ represents the mapping class $\varphi=$ $\boldsymbol{\Theta}_{r} \ldots \boldsymbol{\Theta}_{2} \boldsymbol{\Theta}_{1}$. Clearly, the following proposition is obvious.

Proposition 6. The 3-manifold $M(\mathbf{L})$ is homeomorphic to

$$
M\left(\Theta_{r} \ldots \Theta_{2} \Theta_{1}\right)=M\left(\Theta_{1}, \Theta_{2} \ldots \Theta_{r}\right) .
$$

Actually, given an expression of the mapping class $\varphi$ in Dehn twists

$$
\varphi=\Theta_{r} \ldots \Theta_{2} \Theta_{1},
$$

where $\Theta_{j}$ is a Dehn twist of some circle $\theta_{j}$, for $1 \leq j \leq r$, we have a unique way to construct a link $\mathbf{L}$ of $r$ components to represent $\varphi$ by choosing a sequence of numbers $0<\varepsilon_{1}<\varepsilon_{2}<\cdots<\varepsilon_{r}<1$ and letting $\mathbf{L}_{j}$ be the circle $\left(\theta_{j}, \varepsilon_{j}\right)$ in $\mathbf{F}_{g} \times \varepsilon_{j}$, for all $1 \leq j \leq r$. Thus, when we give an expression of $\varphi$ in Dehn twists, we can always have a framed link $\mathbf{L}$, which represents the map $\varphi$.

\section{The tOPOLOGICAL REALIZATION OF THE ALGEBRAIC PROCESS}

Now we are going to prove Theorem 2. One direction is obviously trivial, that is, the $A$-moves and the $K$-moves leave the 3-manifold unchanged, i.e. $M\left(\mathbf{L}^{i-1}\right)=M\left(\mathbf{L}^{i}\right)$, for all $i$.

The sufficiency of the elementary moves will be proved by using the stable equivalence of the Heegaard decompositions and by using some properties of the mapping class groups.

Let $\varphi$ and $\varphi^{\prime}$ be two mapping classes represented by the framed links $\mathbf{L}$ and $\mathbf{L}^{\prime}$ respectively. If they describe two Heegaard decompositions of the same closed 3-manifold $M=M(\varphi)=M\left(\varphi^{\prime}\right)$. By the Singer stable equivalence theorem [S], we may add enough trivial handles so that both splitting surfaces are 


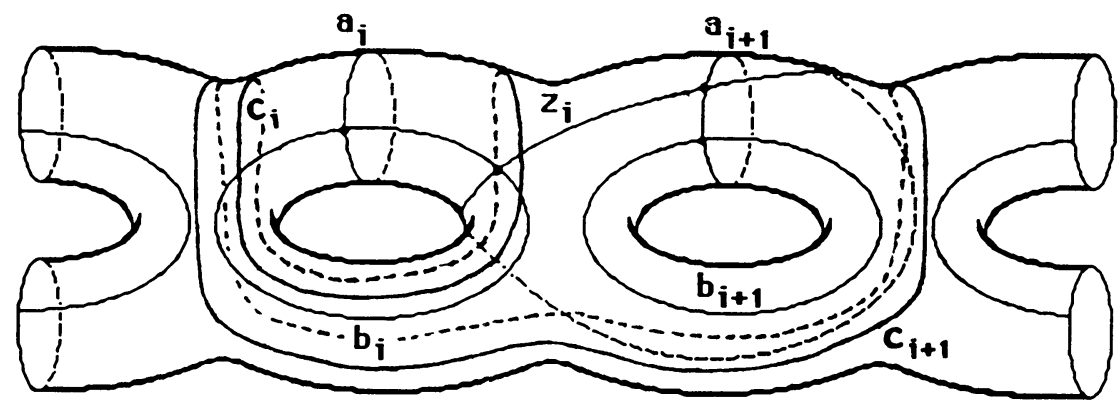

FIGURE 6

isotopic. When we add the trivial handles the twist curves are untouched, thus the new gluing mapping is still represented by the same framed link. Therefore, we may assume that both Heegaard surfaces of the mappings $\varphi$ and $\varphi^{\prime}$ are the same, i.e. $\varphi^{\prime}=f^{\prime} \cdot \varphi \cdot f$, for some $f \in \mathscr{K}_{g}$ and $f^{\prime} \in \mathscr{K}_{g}^{\prime}$, where we denote by $\mathscr{K}_{g}$ and $\mathscr{K}_{g}^{\prime}$ the subgroups of the group $\mathscr{M}_{g}$ consisting of the mapping classes which can be extended to the handlebodies $\mathbf{H}_{g}$ and $\mathbf{H}_{g}^{\prime}$ respectively.

Our proof of Theorem 2 consists of three steps. At first, we change $\mathbf{L}$ by the elementary moves to a link $\mathbf{L}^{1}$, which represents $\varphi^{\prime}$, the mapping class represented by the link $\mathbf{L}^{\prime}$. At second, assuming $\varphi=\varphi^{\prime}$, we change the link $\mathbf{L}$ by the elementary moves to a link $\mathbf{L}^{2}$, which represents $\varphi$ in an expression of the Lickorish generators. Finally, assuming both $\mathbf{L}$ and $\mathbf{L}^{\prime}$ represent $\varphi$ in the Lickorish generators, their expressions can be transformed to each other by using the defining relations of Hatcher-Thurston-Wajnryb's presentation of the mapping class group $\mathscr{M}_{g}$; we will realize these transformations topologically by the elementary moves.

Step 1 . Construct a link $\mathbf{L}^{1}$ representing $\varphi^{\prime}=f^{\prime} \varphi f$ from the link $\mathbf{L}$ by using $D$-moves, $A$-moves and $K$-moves.

This is based on a result in [L].

Theorem L. The group $\mathscr{K}_{g}$ is generated by the Dehn twist $M$ of the meridian circle $a_{1}$ of the solid handlebody $\mathbf{H}_{g}$, and four other elements $\pi, \nu, \tau$ and $\rho$ from $\mathscr{K}_{\mathrm{g}} \cap \mathscr{K}_{\mathrm{g}}^{\prime}$, and the group $\mathscr{K}_{\mathrm{g}}^{\prime}$ is generated by the Dehn twist $\mathbf{L}$ of the meridian circle $b_{1}$ of $\mathbf{H}_{g}^{\prime}$, and the same four elements $\pi, \nu, \tau$ and $\rho$ from $\mathscr{K}_{g} \cap \mathscr{K}_{g}^{\prime}$. Where $\pi=\pi_{1}, \pi_{1}=\left(A_{i} B_{i} A_{i}\right)^{2}$ is a $180^{\circ}$-twist of the ith handle along the waist curve $c_{i}=\left[a_{i}, b_{i}\right], \nu=\nu_{1}, \nu_{i}=\left(A_{i} B_{i} Z_{i} A_{i+1} B_{i+1}\right)^{3}$ is a $180^{\circ}$ twist of the ith and $(i+1)$ th handles along the circle $c_{i}^{\prime}=\left[a_{i}, b_{i}\right]\left[a_{i+1}, b_{i+1}\right]$, $r=\pi_{1}^{2} \nu_{1} \pi_{2}^{2} \nu_{1} \cdots \pi_{g-1}^{2} \nu_{g-1} \pi_{g}^{2}$ rotates the handles, $\rho=A_{1} Z_{1}^{-1} B_{2}$, and $A_{i}, B_{i}$ and $Z_{i}$ are Dehn twists of the curves $a_{i}, b_{i}$ and $z_{i}=\bar{b}_{i+1}\left[b_{i+1}, a_{i+1}\right]\left[b_{i}, a_{i}\right] a_{i}$ respectively as pictured in Figure 6.

Thus, the mapping class $\varphi^{\prime}$ is obtained from $\varphi$ by multiplying some $M$, $\rho, \pi_{i}$ 's and $\nu_{i}$ 's on the right side, and some $L, \rho, \pi_{i}$ 's and $\nu_{i}$ 's on the left side.

Multiplying by $M^{ \pm 1}$ on the right or by $L^{ \pm 1}$ on the left is clearly equivalent to adding a new component $\left(a_{1}^{ \pm 1}, \delta\right)$ or $\left(b_{1}^{ \pm 1}, 1-\delta\right)$, for $\delta>0$ small enough, which is exactly a $D$-move. 


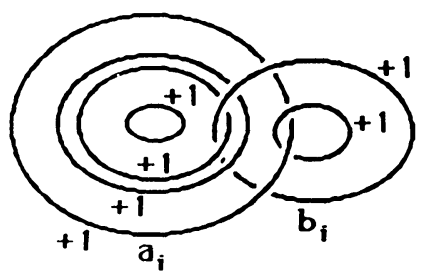

(a)

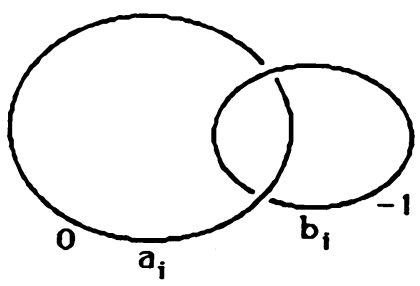

(c)

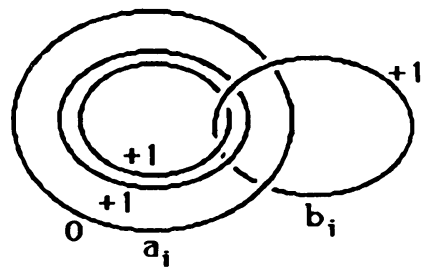

(b)

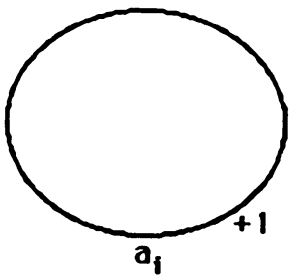

(d)

FIGURE 7

Multiplying by $\rho^{ \pm 1}$ on the right (or similarly, left) is equivalent to adding three components $\left(a_{1}^{ \pm 1}, \delta\right),\left(z_{1}^{ \pm 1}, 2 \delta\right)$ and $\left(b_{2}^{ \pm 1}, 3 \delta\right)$, for some $0<\delta<\frac{1}{3} \varepsilon_{1}$, which is equivalent to one $D$-move that gives $\left(a_{1}^{ \pm 1}, \delta\right)$, and one $A$-move that gives the other two components, since all components are unlinked.

Multiplying by $\pi_{i}^{-1}$ on the right (or similarly, left) is equivalent to adding six components:

$$
\bar{a}_{i} \times\{7 \delta, 9 \delta, 10 \delta, 12 \delta\}, \quad \text { and } \quad \bar{b}_{i} \times\{8 \delta, 11 \delta\},
$$

for some $0<\delta<\frac{1}{12} \varepsilon_{1}$. This can be realized in the following way:

(1) By $A$-moves, we may add the following pairs:

$$
\begin{gathered}
\left(\left(\bar{a}_{i}, 12 \delta\right),\left(a_{i}, \delta\right)\right), \quad\left(\left(\bar{b}_{i}, 11 \delta\right),\left(b_{i}, 2 \delta\right)\right), \quad\left(\left(\bar{a}_{i}, 10 \delta\right),\left(a_{i}, 3 \delta\right)\right), \\
\left(\left(\bar{a}_{i}, 9 \delta\right),\left(a_{i}, 4 \delta\right)\right), \quad\left(\left(\bar{b}_{i}, 8 \delta\right),\left(b_{i}, 5 \delta\right)\right), \quad \text { and } \quad\left(\left(\bar{a}_{i}, 7 \delta\right),\left(a_{i}, 6 \delta\right)\right) .
\end{gathered}
$$

(2) Then, we may remove the components in the levels $\delta, 2 \delta, 3 \delta, 4 \delta, 5 \delta$ and $6 \delta$ by $K$-moves, i.e. by doing the corresponding surgeries, as shown in Figure 7. We first remove $\left(a_{i}, \delta\right)$, none of the circles from the other five levels needs to change. Second we remove $\left(b_{i}, 5 \delta\right)$ and change the label of $\left(a_{i}, 6 \delta\right)$ to 0 . Thirdly we remove $\left(a_{i}, 3 \delta\right)$ and $\left(a_{i}, 4 \delta\right)$ and change the label to $\left(b_{i}, 2 \delta\right)$ to +1 . Fourthly we remove $\left(b_{i}, 2 \delta\right)$ and change the label of $\left(a_{i}, 6 \delta\right)$ back to -1 . And finally we remove $\left(a_{i}, 6 \delta\right)$.

(3) The composition of the $K$-moves in (2) is equal to the mapping class $\pi_{i}$, which is an element of the group $\mathscr{K}_{g} \cap \mathscr{K}_{g}^{\prime}$. Therefore, it is equivalent to an isotopy deformation of the 3-sphere $\mathbf{S}^{3}$ which deforms the thickened surface $\mathbf{F} \times[7 \delta, 1]$ to itself. Now isotopically we deform it back and obtain a link consisting of the old link $\mathbf{L}$ and six more components in the levels $7 \delta, 8 \delta, \ldots, 12 \delta$, which represents the map $\varphi \pi_{i}^{-1}$.

The reverse process gives the way to multiply $\pi_{i}$. 


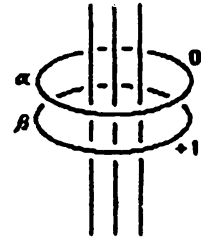

(a)

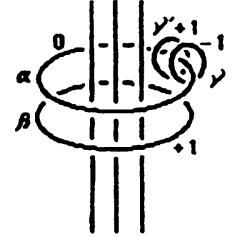

(b)

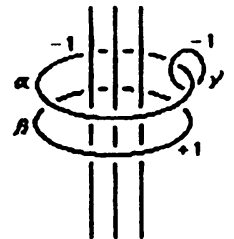

(c)

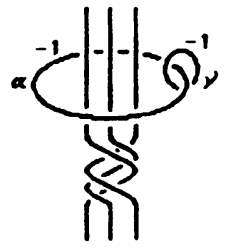

(d)

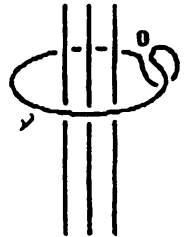

(e)

Figure 8

Finally, multiplying by $\nu_{i}^{-1}$ on the right (or similarly, left) is equivalent to adding fifteen components:

$$
\begin{gathered}
\bar{b}_{i+1} \times\{20 \delta, 25 \delta, 30 \delta\}, \quad \bar{a}_{i+1} \times\{19 \delta, 24 \delta, 29 \delta\}, \quad \bar{z}_{i} \times\{18 \delta, 23 \delta, 28 \delta\}, \\
\bar{b}_{i} \times\{17 \delta, 22 \delta, 27 \delta\}, \quad \text { and } \bar{a}_{i} \times\{16 \delta, 21 \delta, 26 \delta\},
\end{gathered}
$$

for some $0<\delta<\frac{1}{30} \varepsilon_{1}$. This can be realized by the same three steps as we did for $\pi_{i}$.

(1) We add first fifteen pairs of circles

$$
\left\{\mathbf{L}_{31-i}^{\prime}=\left(\bar{x}_{i}(31-i) \delta\right), \mathbf{L}_{i}^{\prime}=\left(x_{i}, i \delta\right)\right\}_{i=1,2, \ldots, 15},
$$

by doing some $A$-moves, where $x_{i}=a_{i}$ if $i=5,10,15, x_{i}=b_{i}$ if $i=$ $4,9,14, x_{i}=z_{i}$ if $i=3,8,13, x_{i}=b_{i+1}$ if $i=2,7,12$ and $x_{i}=a_{i+1}$ if $i=1,6,11$.

(2) In order to remove the components $\mathbf{L}_{1}^{\prime}, \mathbf{L}_{2}^{\prime}, \ldots, \mathbf{L}_{15}^{\prime}$, we need the following lemma:

Lemma 7. We may simply insert or eliminate an unknotted component with label \pm 1 by using some $K$-moves and A-moves if there is some component with label 0 parallel to it.

Proof. As shown in Figure 8(a), let $\beta$ be an unknotted component with label \pm 1 , and let $\alpha$ be a component parallel to $\beta$ with label 0 . We first insert a pair $\gamma$ and $\gamma^{\prime}$ with the labels $\mp 1$ and \pm 1 around the circle $\alpha$ respectively by doing an $A$-move (Figure 8(b)). Next we do the $K$-move to remove the component $\gamma^{\prime}$, that changes the label of $\alpha$ into $\mp 1$ (Figure 8(c)). Then similarly we do the $K$-moves to eliminate the components $\beta$ (Figure 8(d)) and $\alpha$ (Figure 8(e)). Thus the lemma is done.

Now consider the link $\left\{\mathbf{L}_{k}^{\prime}\right\}_{1 \leq k \leq 15}$ pictured in Figure $9(\mathbf{a})$. We first eliminate the components $\mathbf{L}_{5}^{\prime}, \mathbf{L}_{14}^{\prime}, \mathbf{L}_{2}^{\prime}$ and $\mathbf{L}_{11}^{\prime}$ by doing some $K$-moves (Figure 9 (b)) that change the labels of $\mathbf{L}_{4}^{\prime}, \mathbf{L}_{15}^{\prime}, \mathbf{L}_{1}^{\prime}$ and $\mathbf{L}_{12}^{\prime}$ into 0 . Second we eliminate the components $\mathbf{L}_{10}^{\prime}, \mathbf{L}_{13}^{\prime}, \mathbf{L}_{3}^{\prime}$ and $\mathbf{L}_{6}^{\prime}$ by Lemma 3.2 (Figure $9(\mathrm{c})$ ). Thirdly we eliminate the components $\mathbf{L}_{9}^{\prime}$ and $\mathbf{L}_{7}^{\prime}$ by doing some $K$-moves (Figure $9(\mathbf{d})$ ) that change the labels of $\mathbf{L}_{15}^{\prime}$ and $\mathbf{L}_{1}^{\prime}$ to be $-1 s$. Fourthly we eliminate the components $\mathbf{L}_{15}^{\prime}$ and $\mathbf{L}_{1}^{\prime}$ by doing some $K$-moves (Figure $9(e)$ ) that change the labels of $\mathbf{L}_{4}^{\prime}$ and $\mathbf{L}_{12}^{\prime}$ to be -1 . Fifthly we eliminate the components $\mathbf{L}_{4}^{\prime}$ and $\mathbf{L}_{12}^{\prime}$ by some $K$-moves (Figure $9(\mathrm{f})$ ) that change the label of $\mathbf{L}_{8}^{\prime}$ to be -1 . And finally do the $K$-move to remove $\mathbf{L}_{8}^{\prime}$.

(3) As we did for $\pi_{i}^{-1}$, deform the link back to the original embedding by an isotopy of the 3 -sphere $\mathbf{S}^{3}$, since $\pi_{i} \in \mathscr{K}_{g} \cap \mathscr{K}_{g}^{\prime}$. 


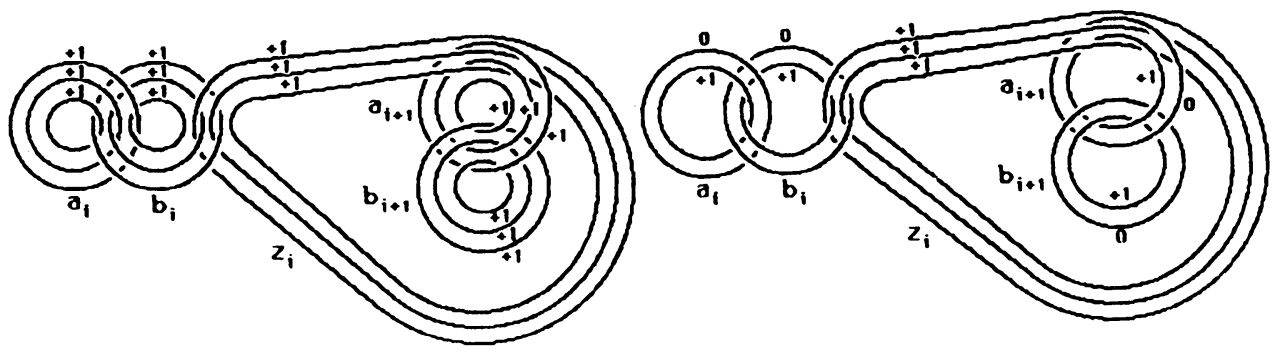

(a)

(b)

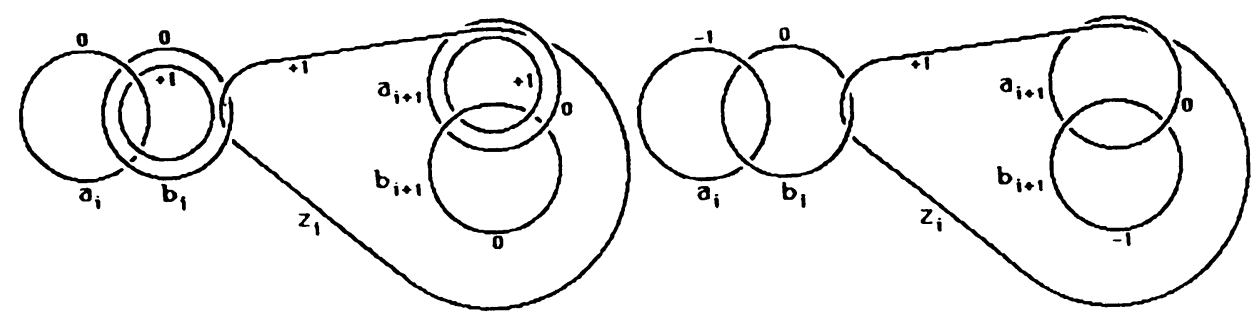

(c)

(d)

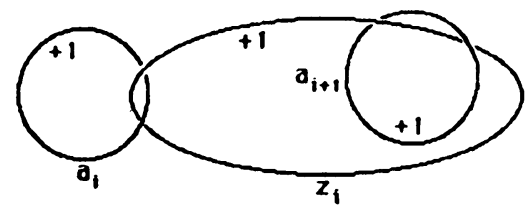

(e)

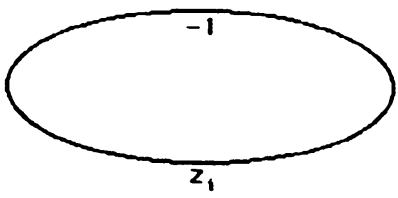

(f)

FIGURE 9

The reverse process gives the way to multiply $\nu_{i}$.

Repeatedly using the above process, Step 1 can be accomplished clearly. And we may assume $\varphi=\varphi^{\prime}$ from now on.

Step 2. We can change the links $\mathbf{L}$ and $\mathbf{L}^{\prime}$ into a form that each component corresponds to some twist curve of some Lickorish generator generated by using some $A$-moves and $K$-moves.

For $\mathbf{L}=\left\{\mathbf{L}_{j}\right\}_{1 \leq j \leq r}$, the representation of $\varphi$ given by $\mathbf{L}$ corresponds to an expression of $\varphi$ in Dehn twists:

$$
\varphi=\Theta_{r} \cdots \Theta_{j} \cdots \Theta_{1}
$$

where $\boldsymbol{\theta}_{j}$ is the Dehn twist of the curve $\theta_{j}$, which represents the component $\mathbf{L}_{j}=\left(\theta_{j}, \varepsilon_{j}\right)$, for $0<\varepsilon_{1}<\cdots<\varepsilon_{r}<1$.

Recall that, for each $j=1, \ldots, r$, we may assume that $\theta_{j}$ intersects either some $a_{i}$ or some $b_{i}$ transversely at exactly one point. Indeed, as we did in $\S 2$, we may assume that each of the original $\theta_{j}$ 's from $\mathbf{L}$ intersected some $b_{i}$ 

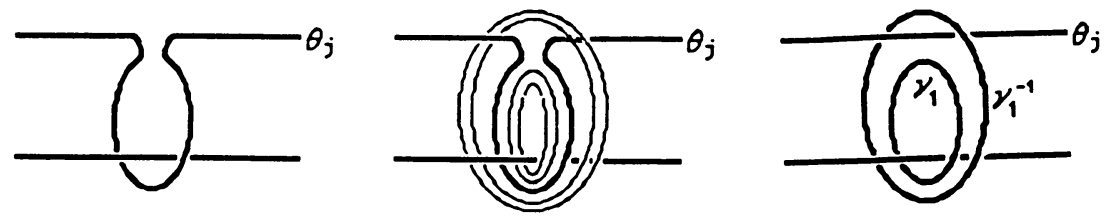

FIGURE 10

transversely at exactly one point. And all new added components by Step 1 also have this property, because they represent Lickorish generators. Thus the closed curve $\theta_{j}$ is not null-homologous in the closed surface $\mathbf{F}_{g}$. Therefore, the Dehn twist $\boldsymbol{\Theta}_{j}$ is conjugate of $L$, the Dehn twist of the circle $b_{1}$, and $\boldsymbol{\Theta}_{j}$ admits an expression in the Lickorish generators of the form

$$
\boldsymbol{\Theta}_{j}=\Gamma_{1} \Gamma_{2} \cdots \Gamma_{l} L \Gamma_{l}^{-1} \cdots \Gamma_{2}^{-1} \Gamma_{1}^{-1},
$$

where each $\Gamma_{i}$ is a Lickorish generator. We will realize this expression topologically by doing some $A$-moves and $K$-moves inductively on the number $l$.

Actually, let $\Theta_{j}^{1}=\Gamma_{2} \cdots \Gamma_{l} L \Gamma_{l}^{-1} \cdots \Gamma_{2}^{-1}$, which is a Dehn twist of some curve $\theta_{j}^{\prime}$, and $\gamma_{1}$ and $\gamma_{1}^{-1}$ denote the twist curves representing the Lickorish generators $\Gamma_{1}$ and $\Gamma_{1}^{-1}$, and let $\delta$ be a small number with

$$
0<2 \delta<\max \left\{\varepsilon_{j}-\varepsilon_{j-1}, \varepsilon_{j+1}-\varepsilon_{j}\right\}
$$

(Figure 10). We can replace the component $\mathbf{L}_{j}=\left(\theta_{j}, \varepsilon_{j}\right)$ by three components $\mathbf{L}_{j 1}=\left(\gamma_{1}, \varepsilon+2 \delta\right), \mathbf{L}_{j}^{\prime}=\left(\theta_{j}^{\prime}, \varepsilon_{j}\right)$ and $\mathbf{L}_{j 2}=\left(\gamma^{-1}, \varepsilon_{j}-2 \delta\right)$ in the following way:

(1) Use two $A$-moves to insert two pairs of $\left(\gamma, \gamma^{-1}\right)$; one pair consists of $\mathbf{L}_{j 1}=\left(\gamma_{1}, \varepsilon+2 \delta\right)$ and $\mathbf{L}_{j 1}^{\prime}=\left(\gamma_{1}^{-1}, \varepsilon+\delta\right)$, and the other consists of $\mathbf{L}_{j 2}^{\prime}=$ $\left(\gamma_{1}, \varepsilon-\delta\right)$ and $\mathbf{L}_{j 2}=\left(\gamma_{1}^{-1}, \varepsilon-2 \delta\right)$, i.e. the expression becomes $\Gamma_{1} \Gamma_{1}^{-1} \boldsymbol{\Theta}_{j} \Gamma_{1} \Gamma_{1}^{-1}$.

(2) Then use two $K$-moves to eliminate the components $\mathbf{L}_{j 1}^{\prime}=\left(\gamma_{1}^{-1}, \varepsilon+\delta\right)$ and $\mathbf{L}_{j 2}^{\prime}=\left(\gamma_{1}, \varepsilon-\delta\right)$, i.e. replace $\Gamma_{1}^{-1} \Theta_{j} \Gamma_{1}$ by $\Theta_{j}^{\prime}$.

Repeatedly using this method until $\Theta_{J}^{\prime}=L$, the old component $\mathbf{L}_{j}$ has been replaced by $2 l+1$ components which represent $\Gamma_{1}, \Gamma_{2}, \ldots, \Gamma_{l}, L, \Gamma_{l}^{-1}, \ldots$, $\Gamma_{2}^{-1}, \Gamma_{1}^{-1}$ respectively.

Doing the same process for each $\Theta_{j}$, the link $\mathbf{L}$ can be transformed into the required form. The same argument can be done for the link $\mathbf{L}^{\prime}$ which completes Step 2.

Step 3. If $\mathbf{L}$ and $\mathbf{L}^{\prime}$ are both formed by the twist curves of the Lickorish generators and both represent the same mapping class $\varphi^{\prime}=\varphi$, then they are related by some elementary moves.

Since both the links $\mathbf{L}$ and $\mathbf{L}^{\prime}$ are formed by the twist curves of the Lickorish generators and both represent the same mapping class $\varphi^{\prime}=\varphi$, i.e. they give two different expressions of $\varphi$ in the Lickorish generators. Hence, they must be related by finitely many insertions or eliminations of either a word of the form $\Delta^{ \pm 1} \Delta^{\mp 1}$, for $\Delta$ a Lickorish generator, or a defining relation of some presentation of the surface mapping class group $\mathscr{M}_{g}$. So now, all we need is to realize this algebraic process by topological moves.

Claim 1. An insertion of a word of type $\Delta^{ \pm 1} \Delta^{\mp 1}$ is equivalent to an $A$-move.

This is obvious from the construction. 


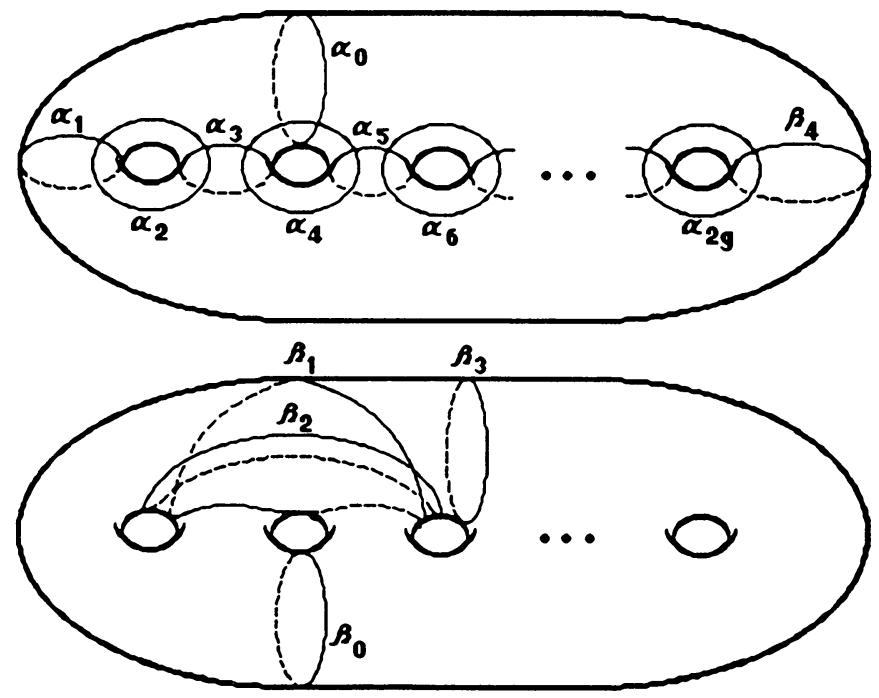

FIGURE 11

Claim 2. An elimination of a relation in Hatcher-Thurston-Wajnryb's presentation of the surface mapping class group $\mathscr{M}_{g}$, for some $g \geq 3$, is equivalent to a composition of $K$-moves.

We recall the presentation first (cf. [B or W]):

Theorem W (Hatcher-Thruston-Wajnryb). The mapping class group $\mathscr{M}_{g}$ of the closed orientable surface $\mathbf{F}_{g}$, for $g \geq 3$, is generated by the Dehn twists $A_{0}, A_{1}$, $\ldots, A_{2 g}$ of the curves $\alpha_{0}, \alpha_{1}, \ldots, \alpha_{2 g}$ as drawn in Figure 11 respectively, and is presented by the relations:

$$
\begin{array}{ll}
A_{i} A_{j}=A_{j} A_{i}, & \text { if } \alpha_{i} \cap \alpha_{j}=\varnothing, \\
A_{i} A_{j} A_{i}=A_{j} A_{i} A_{j}, & \text { if } \#\left(\alpha_{i} \cap \alpha_{j}\right)=1, \\
A_{0} B_{0}=\left(A_{1} A_{2} A_{3}\right)^{4}, & \\
A_{0} B_{1} B_{2}=A_{1} A_{3} A_{5} B_{3}, & \\
B_{4}=G B_{4} G^{-1}, &
\end{array}
$$

where (f) $B_{i}=F_{i} A_{0} F_{i}^{-1}$ is the Dehn twist of the curve $\beta_{i}$ pictured in Figure 11, for $i=0,1,2,3,4$, and $G$ and $F_{i}$ 's are given words in $A_{i}$ 's.

To eliminate a part of link given by a defining relation, is equivalent to doing the corresponding surgeries on this part, since the composition of them gives the identity. So, what we need is to put the components of that part in a good order, such that we may do surgeries on these components in that order with the property that, in each step the surgery is a \pm 1 -surgery on an unknotted component, i.e. a $K$-move.

Now we discuss the cases separately.

Cases (a) and (d). In these cases, since the linking number of any two components is zero, when we do a $K$-move on one component the other components remain unknotted with the original labels. Thus, we may remove them in an arbitrary order. 


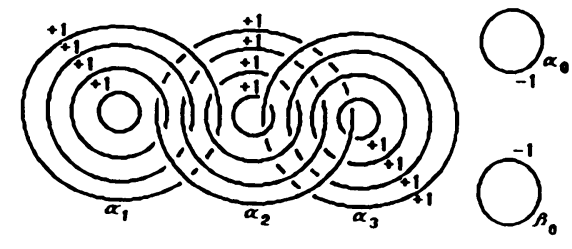

(a)

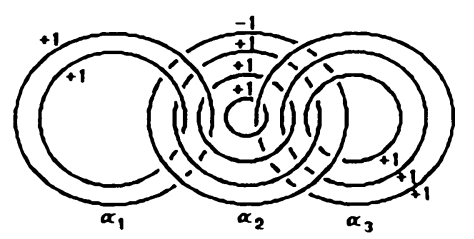

(c)

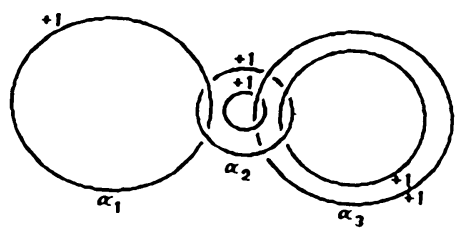

(e)

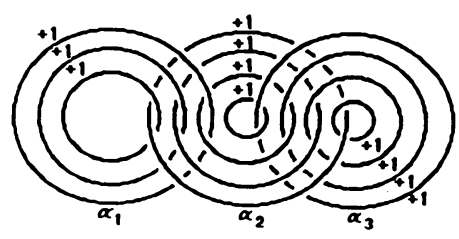

(b)

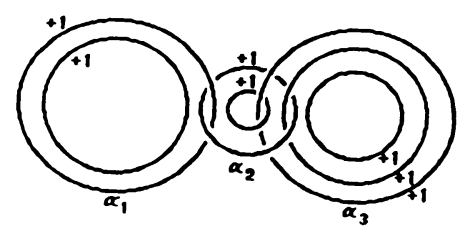

(d)

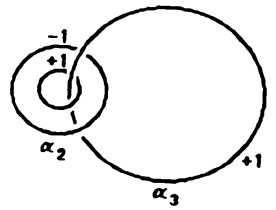

(f)

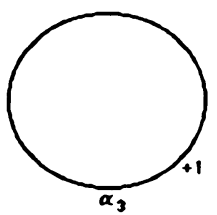

(g)

FIGURE 12

Cases (b), (e) and (f). We may unify these cases in the following form

$$
B^{-1} \Theta_{n} \cdots \Theta_{2} \Theta_{1} A \Theta_{1}^{-1} \Theta_{2}^{-1} \cdots \Theta_{n}^{-1}=1,
$$

where $\boldsymbol{\Theta}_{j}=A_{i_{j}}$ for some $i_{j}$, for $j=1,2, \ldots, n$. We denote $\theta_{i}, \bar{\theta}_{i}, \alpha$ and $\beta$ the components representing $\Theta_{i}, \Theta_{i}^{-1}, A$ and $B^{-1}$ respectively, for $i=1,2, \ldots, n$. And we may order them in the following way:

$$
\theta_{1}, \bar{\theta}_{1}, \theta_{2}, \bar{\theta}_{2}, \ldots, \theta_{n}, \bar{\theta}_{n}, \alpha, \beta \text {. }
$$

In fact, when we remove $\theta_{1}, \bar{\theta}_{1}$ remains the same. After we remove both $\theta_{1}$ and $\bar{\theta}_{1}$, all components remain the same as before except the component $\alpha$ is possibly changed, which represent $\theta_{1} A \Theta_{1}^{-1}$ now. Hence, we repeat the process for $\theta_{2}$, and $\bar{\theta}_{2}$, and so on until we remove $\theta_{n}$ and $\bar{\theta}_{n}$. Now the link contains only two components, one is $\beta$, representing $B^{-1}$, which had never been changed, and the other one is $\alpha$, which represents $\Theta_{n} \cdots \Theta_{2} \Theta_{1} A \Theta_{1}^{-1} \Theta_{2}^{-1} \cdots \Theta_{n}^{-1}=B$. Thus $\alpha$ must be a component parallel to $\beta$ with the opposite label, since any two curves in the surface representing the same Dehn twist must be isotopic. It implies that $\alpha$ is \pm 1 -labeled, unknotted, and unlinked with $\beta$. 

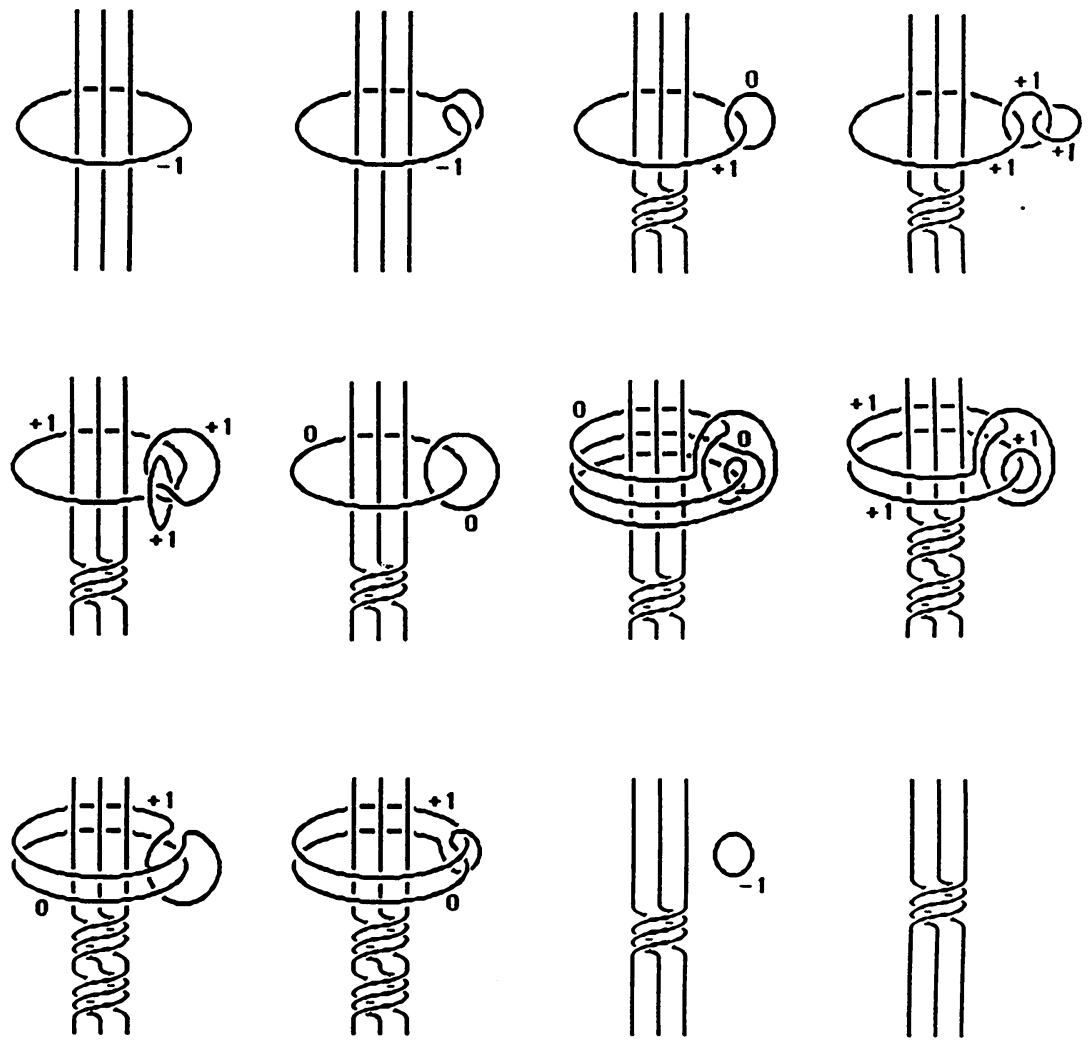

FIGURE 13

Case (c). As drawn in Figure 12(a), we have 14 components:

$\alpha=\left(\alpha_{0}^{-1}, \varepsilon+13 \delta\right), \quad \beta=\left(\beta_{0}^{-1}, \varepsilon+14 \delta\right), \quad$ and $\quad \alpha_{i}^{j}=\left(\alpha_{i}, \varepsilon+(3 j-i+1) \delta\right)$, for $i=1,2,3$ and $j=1,2,3,4$, in the levels $\varepsilon+\delta, \varepsilon+2 \delta, \ldots, \varepsilon+14 \delta$, for some $0<\varepsilon, \delta<1$, which represent $A_{0}^{-1}, B_{0}^{-1}$, and $A_{i}, i=1,2,3$, respectively. We will remove the components in the following order:

$$
\alpha, \beta, \alpha_{1}^{1}, \alpha_{1}^{2}, \alpha_{3}^{1}, \alpha_{2}^{1}, \alpha_{2}^{2}, \alpha_{1}^{3}, \alpha_{3}^{2}, \alpha_{1}^{4}, \alpha_{3}^{3}, \alpha_{2}^{3}, \alpha_{2}^{4}, \alpha_{3}^{4} .
$$

Precisely, since $\alpha, \beta$, and $\alpha_{1}^{1}$ are not linked with any component of the sublink which represents relation (c), we may first do the $K$-moves to remove them (Figure 12(b)).

Next we do the $K$-moves to eliminate $\alpha_{1}^{2}$ and $\alpha_{3}^{1}$, which switch only the label of the component $\alpha_{2}^{1}$ from +1 to -1 (Figure 12(c)). Now the components $\alpha_{2}^{1}$ and $\alpha_{2}^{2}$ become two parallels with the oppose labels, we eliminate them by an $A^{-1}$-move (Figure 12(d)). And now we have unlinked components, $\alpha_{1}^{3}$ and $\alpha_{3}^{2}$, and remove them by doing some $K$-moves (Figure 12(e)). After then we remove the components $\alpha_{1}^{4}$ and $\alpha_{3}^{3}$ which only switch the sign of $\alpha_{2}^{3}$ (Figure 12(f)). Then we remove $\alpha_{2}^{3}$ and $\alpha_{2}^{4}$ by doing an $A^{-1}$-move (Figure 12(g)). Finally do a $K$-move to remove $\alpha_{3}^{4}$.

.As we pointed out in the last section, a $K^{-1}$-move is a composition of an $A$-move and a $K$-move. And an $A^{-1}$-moves is a composition of two $K$-moves. 
Hence, an inverse process of Claim 2 or Claim -an elimination of a word of type $\Delta^{ \pm 1} \Delta^{\mp 1}$ or an insertation of a relation in Hatcher-Thurston-Wajnryb's presentation of the surface mapping class group $\mathscr{M}_{g}$, for some $g \geq 3$-is also equivalent to some composition of the $A$-moves and $K$-moves. Therefore, we have a complete topological realization of the algebraic process, which finishes our proof of Theorem 1.2.

Before ending the paper, we present an interesting fact that we learned from Professor R. Lickorish, which is a little bit stronger than Theorem 1.

Theorem 8. The integer framed links determine the same 3-manifold if and only if they are related by +1 -labeled $K^{ \pm 1}$-moves and -1-labeled $D^{ \pm 1}$-moves.

Proof. All we need to show is that, a -1-labeled $K^{ \pm 1}$-move is a composition of some +1 -labeled $K^{ \pm 1}$-moves and some -1 -labeled $D^{ \pm 1}$-moves. This is clearly shown in Figure 13.

\section{ACKNOWLEDGMENT}

This paper is a chapter of my Ph.D. Thesis, I would like to thank my advisor Professor John Hempel for the years of help and instruction.

\section{REFERENCES}

[B] J. S. Birman, Mapping class groups of surfaces, Braids, Contemp. Math., vol. 78, Amer. Math. Soc., Providence, R.I., 1986, pp. 13-43.

[FR] R. Fenn and C. Rourke, On Kirby's calculus of links, Topology 18 (1979), 1-15.

[K] R. Kirby, $A$ calculus for framed links in $\mathbf{S}^{3}$, Invent. Math. 45 (1978), 35-56.

[Li] W. B. R. Lickorish, A representation of orientable combinatorial 3-manifolds, Ann. of Math. (2) 76 (1962), 531-540.

[L] N. Lu, Homeomorphisms of a handlebody and Heegaard splittings of the 3-sphere $\mathbf{S}^{3}$, Topology Proc. 13 (1988), 325-350.

[R] D. Rolfsen, Rational surgery calculus: extension of Kirby's theorem, Pacific J. Math. 110 (1984), 377-386.

[W] B. Wajnryb, A simple presentation for the mapping class group of an orientable surface, Israel J. Math. 45 (1983), 157-174.

[Wa] A. H. Wallace, Modifications and cobounding manifolds, Canad. J. Math. 12 (1960), 503528.

Department of Mathematics, Rice University, Houston, Texas 77251

Current address: Iterated Systems, Inc., 5550-A Peachtree Parkway, Norcross, Georgia 30092 